\title{
Significance of SALL4 as a drug-resistant factor in lung cancer
}

\author{
NOZOMI YANAGIHARA ${ }^{1}$, DAISUKE KOBAYASHI ${ }^{1}$, KAGEAKI KURIBAYASHI $^{1}$, \\ MAKI TANAKA $^{1}$, TADASHI HASEGAWA ${ }^{2}$ and NAOKI WATANABE ${ }^{1}$ \\ Departments of ${ }^{1}$ Clinical Laboratory Medicine and ${ }^{2}$ Surgical Pathology, \\ Sapporo Medical University School of Medicine, Sapporo 060-8543, Japan
}

Received October 15, 2014; Accepted December 4, 2014

DOI: 10.3892/ijo.2015.2866

\begin{abstract}
We examined first evidence for the significance of SALL4, a transcription factor essential for embryonic development and the self-renewal of embryonic stem (ES) cells, as a natural resistance factor against anticancer drugs in lung cancer. To determine the significance of SALL4 expression in lung cancer cells, small inhibitory RNA (siRNA) against SALL4 was transduced into A549 and SBC-3 cells, resulting in increased sensitivity towards anticancer drugs [cisplatin (CDDP), carboplatin (CBDCA), and paclitaxel (PTX)]. SALL4 cancer tissues from 31 lung cancer patients were used to assess clinical significance. The analysis showed differences in SALL4 expression corresponding to the therapeutic outcomes. SALL4 expression measured before adjuvant chemotherapy was significantly higher in the patients showing recurrence of cancer than in the disease-free patients. In addition, the period until recurrence was shorter in the patients showing high SALL4 expression. These results indicate that SALL4 overexpression acts as a natural resistance factor and may be involved in the recurrence of lung cancer after adjuvant chemotherapy.
\end{abstract}

\section{Introduction}

The prognosis for lung cancer continues to remain poor, and in stage I-III cases, this is largely due to drug resistance leading to recurrence after adjuvant chemotherapy. Energy-dependent rapid drug efflux and multidrug-resistant molecules are known as factors involved in the resistance of several types of cancer cells; however, drug retention does not always correlate with its cytotoxicity, and therefore, there have been many cases in which the mechanism of resistance remains unknown. In addition, excluding specific gene mutations for molecules such as epidermal growth

Correspondence to: Professor Naoki Watanabe, Department of Clinical Laboratory Medicine, Sapporo Medical University School of Medicine, South-1, West-16, Chuo-Ku, Sapporo 060-8543, Japan E-mail: watanabn@sapmed.ac.jp

Key words: SALL4, measurement, lung cancer, drug resistance, recurrence, prediction factor receptor (EGFR), K-ras, and EML/ALK4 in molecular-targeted therapy, markers to determine drug sensitivity and to predict recurrence after chemotherapy are yet to be identified. Therefore, it is urgent to seek universal markers in which constitutive expression reflects drug resistance in lung cancer.

Cancer cells have the capacity for self-renewal through uncontrolled proliferation and dedifferentiation, similar to embryonic stem (ES) cells (1). Several molecules that are expressed during early embryonic development are important in the maintenance of mouse ES cell self-renewal (2-5). These molecules also generate and maintain the ability of induced pluripotent stem (iPS) cells to self-renew in mice and humans (6-8). Of these molecules, Sall4 is a key factor in maintaining the undifferentiated state and cell proliferation (9). Knockdown of Sall4 expression leads to catastrophic ES-cell proliferation, and Sall4-knockout mice do not survive until embryonic day 7 (9).

Sall4 is the mouse homolog of the Drosophila homeotic gene spalt (sal) and is required for the early development of the posterior head and anterior tail of Drosophila (10). Sal also regulates pattern formation and cell fate decisions in the wing disc, trachea, and sensory organs. Mutations in the human homolog SALL4 are known to cause Okihiro syndrome (Duane-radial ray syndrome), characterized by limb deformities and loss of eye movement $(11,12)$. In some cases, anomalies of the rectum, ear, heart, and kidney are also observed. The SALL4/Sall4 gene is constitutively expressed in human and mice CD34-positive hematopoietic stem cells (13). Interestingly, the overexpression of Sall4 leads to leukemogenesis by increasing the number of leukemic cells with markers for stem cells in $50 \%$ of transgenic mice (13). In fact, SALL4 is overexpressed in various types of human hematopoietic malignancies, such as acute myelocytic and lymphocytic leukaemia $(14,15)$.

Moreover, SALL4 upregulates the expression of the oncogene Bmi-1 in human hematopoietic stem cells and leukemic cells (16). Bmi-1 activates telomerase reverse transcriptase, thereby inducing telomerase activity and leading to the transformation of human non-cancerous epithelial cells (17). Bmi-1 also inhibits the function of INK4a/ARF, usually by disturbing cyclin-dependent kinases 2, 4, and 6 (18), indicating that Bmi-1 expression leads to the progression of the cell cycle from G1 to the S phase. Consequently, SALL4 expression may lead to transformation of non-cancerous cells. 
Previously, we found that SALL4 mRNA expression is significantly higher in cancerous cells than in the non-cancerous tissues of breast and lung cancer patients $(19,20)$. SALL4 is already highly expressed at the early-stage IA, especially in lung cancer, suggesting that it has an essential role in carcinogenesis (20). SALL4 expression may characterize a feature of drug resistance, which has been observed in the stem cell population; evidence for this hypothesis has been demonstrated in a recent study using leukemic cells (21). However, the role of SALL4 in drug resistance has not yet been reported in other cancers. Furthermore, the relationship between SALL4 expression and prognosis, especially for recurrence, remains unclear. Therefore, in this study, we examined the effect of alteration of $S A L L 4$ expression on resistance to anticancer drugs and analyzed the relationship between the expression levels of SALL4 before chemotherapy and the recurrence of lung cancer.

\section{Materials and methods}

Patients and tissue samples. Paraffin-embedded tissue samples from 31 lung cancers (13 adenocarcinomas, 14 squamous cell carcinomas, and 4 small-cell lung cancers) were obtained after surgery. The tissue samples were stained with hematoxylin/eosin and reviewed by experienced pathologists. Clinicopathological factors and clinical stages were evaluated on the basis of the tumor-node-metastasis staging system.

Anticancer drugs. Cisplatin (CDDP), carboplatin (CBDCA), and paclitaxel (PTX) were purchased from Sigma-Aldrich (St. Louis, MO, USA). Each drug was dissolved in water or DMSO, and small aliquots at high concentration were frozen at $-40^{\circ} \mathrm{C}$ until use.

Cell culture. The human lung cancer cell line A549 was cultured in Eagle's minimal essential medium (MEM) and non-essential amino acid solution (both from Sigma-Aldrich Japan K.K., Tokyo, Japan) supplemented with $10 \%$ heat-inactivated fetal bovine serum (FBS) (Invitrogen Life Technologies, Carlsbad, CA, USA). SBC-3 was cultured in MEM (Sigma-Aldrich) supplemented with $10 \%$ heat-inactivated FBS (Invitrogen Life Technologies). The cells were grown at $37^{\circ} \mathrm{C}$ in a humidified atmosphere of $5 \% \mathrm{CO}_{2}$.

RNA extraction and quantification of SALL4 mRNA expression. Total RNA from the lung cancer samples in formalin-fixed, paraffin-embedded tissues or from cancer cell lines was extracted using an RNeasy FFPE isolation kit or RNeasy Plus Mini kit (both from Qiagen, Tokyo, Japan) according to the manufacturer's instructions. The expression of SALL4 mRNA was determined using quantitative reverse transcription-polymerase chain reaction (RT-qPCR) according to the experimental procedure described in our earlier report $(19,20)$.

Western blotting. After the various treatments indicated in each figure, the cells were harvested in a lysis buffer $(50 \mathrm{mM}$ Tris- $\mathrm{HCl}, \mathrm{pH}$ 8.0, $150 \mathrm{mM} \mathrm{NaCl}, 5 \mathrm{mM}$ EDTA) containing a protease inhibitor cocktail (Sigma-Aldrich), sonicated for $30 \mathrm{sec}$, and centrifuged at $15,000 \mathrm{rpm}$ for $5 \mathrm{~min}$. The supernatants were mixed with 1:2 volumes of sample buffer

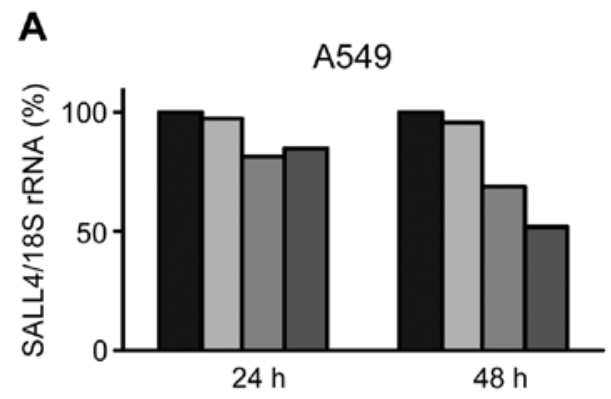

B $\quad \mathrm{SBC}-3$

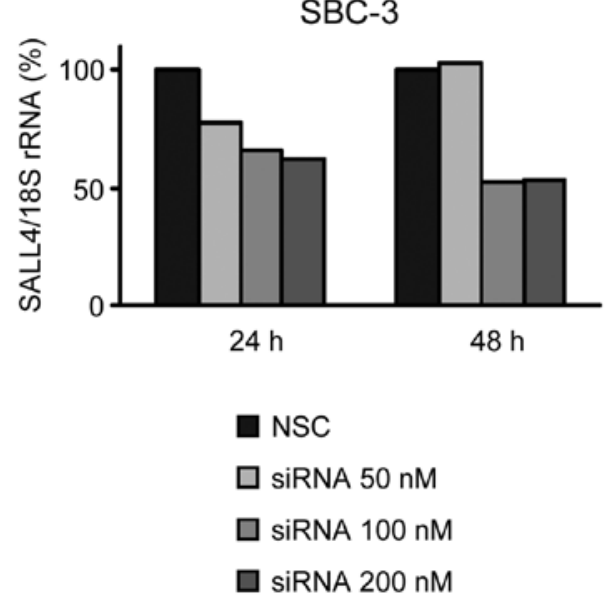

Figure 1. The effect of SALL4 small inhibitory RNA (siRNA) on SALL4 mRNA in (A) A549 and (B) SBC-3 cells. The cells were transduced with non-silencing control (NSC) RNA or several concentrations of siRNA, and total RNA was extracted after 24 and $48 \mathrm{~h}$. Twenty nanograms of total RNA were used for the quantitative measurement of SALL4 mRNA expression by TaqMan RT-PCR. The expression level of SALL4 mRNA in the transduced cells is presented relative to that of $18 \mathrm{~S}$ rRNA. The mean values for triplicate measurements were converted to a percentage relative to expression in NSC-transduced cells.

(red loading buffer reagent; New England Biolabs, Ipswich, MA, USA) and boiled for $2 \mathrm{~min}$, and separated on a $4-20 \%$ Tris-glycine gradient gel (Invitrogen Life Technologies) under denaturing conditions. The proteins were electroblotted onto a nitrocellulose membrane and reacted with antibody against SALL4 (ab29112; Abcam, Tokyo, Japan) or $\beta$-actin (monoclonal AC-15; Sigma-Aldrich). Then, each protein was detected using ECL Prime Western Blotting Detection Reagent (GE Healthcare Japan Corp., Tokyo, Japan), according to the manufacturer's instructions. The bands were visualized and imaged using ChemiDoc XRS Plus (Bio-Rad, Hercules, CA, USA).

Microarray analysis. Total RNA from gene-transduced cells was prepared and subjected to industrial analysis. Quality control was performed, and global gene expression profiling was carried out by Takara Bio, Inc. (Shiga, Japan) using the Agilent SurePrint G3 Human GE 8x60K Microarrays, as per the Agilent One-Color Microarray-Based Gene Expression Analysis protocol (Agilent Technologies, Inc., Santa Clara, CA, USA). The slides were scanned using an Agilent Technologies Microarray Scanner, and the image data were processed using Agilent Feature Extraction software, version 10.10.1.1. The 

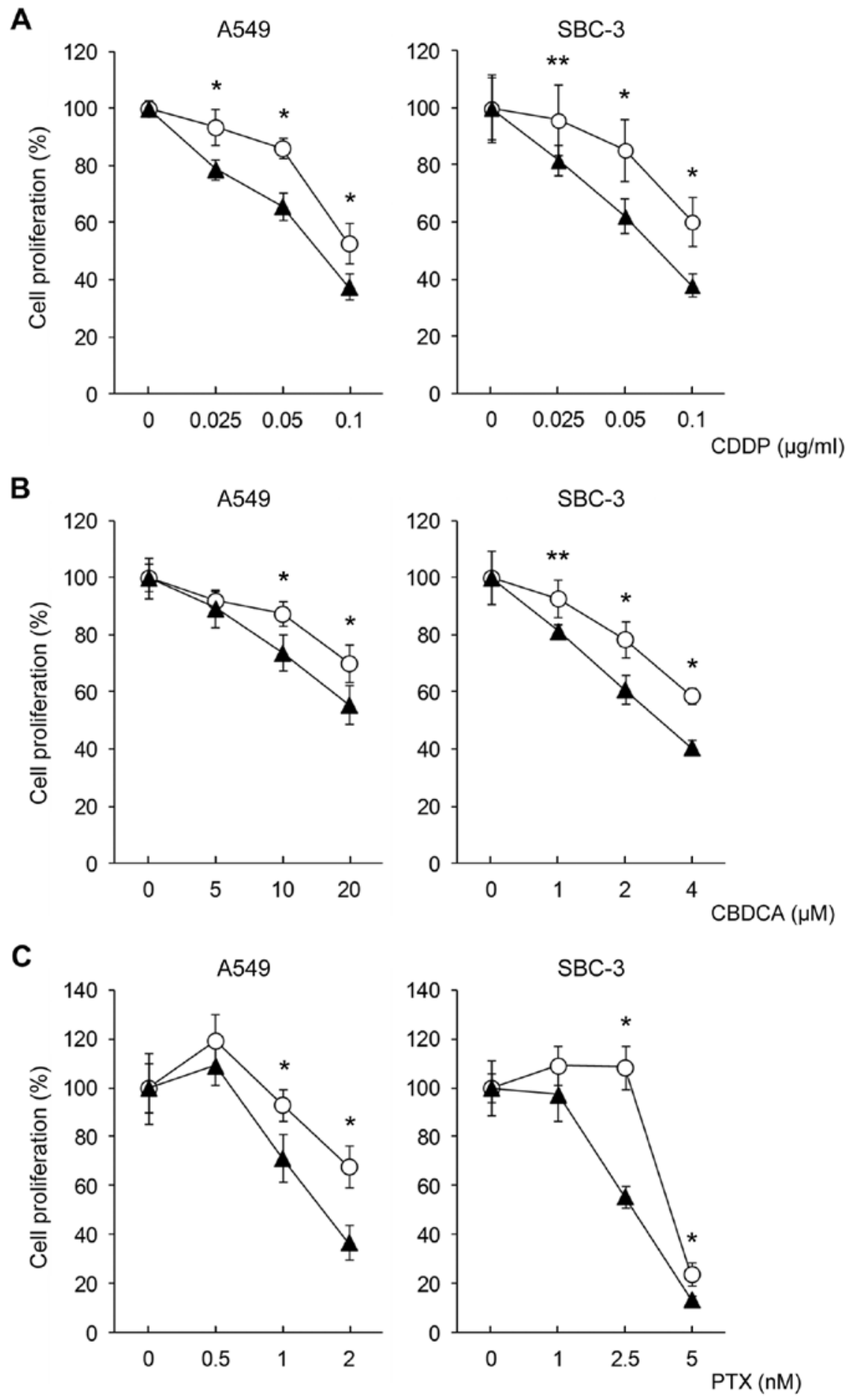

Figure 2. Alteration of drug sensitivity in A549 and SBC-3 cells by SALL4 small inhibitory RNA (siRNA) transduction. The cells were transduced with non-silencing control (NSC) RNA (open circle) or siRNA (closed triangle), and treatment with anticancer drugs (A) cisplatin (CDDP), (B) carboplatin (CBDCA) or (C) paclitaxel (PTX) was started after $48 \mathrm{~h}$ of transduction. The cells were cultured for 1-4 days, and the alteration of the cell proliferation was assessed by the ATP assay. The data represent the mean \pm SD for five independent measurements at day 4 after the treatment by each anticancer drug. ${ }^{*} \mathrm{P}<0.01$ and ${ }^{* *} \mathrm{p}<0.05$ by paired Student's t-test.

gene expression levels were compared after global normalization. The data have been deposited at the National Center for Biotechnology Information (NCBI) Gene Expression Omnibus with the accession no. GSE56595.

Measurement of cell viability. Cells transduced with small inhibitory RNA (siRNA) were plated in 96-well plates at a density of 500-1,000 cells/well in the media supplemented with $10 \%$ FBS. The cells were allowed to adhere for $24 \mathrm{~h}$, and anticancer drugs were added after an additional $24 \mathrm{~h}$ incubation. The culture plate was subjected to the Cell Titer-Glo ${ }^{\mathrm{TM}}$ Luminescent Cell Viability assay (Promega Corp., Madison, WI, USA), according to the manufacturer's instructions. The level of ATP-derived luminescent signal, which correlates with the number of viable cells, was measured using a Veritas ${ }^{\mathrm{TM}}$ Microplate Luminometer (Promega Corp.). 
Cell cycle analysis. Cells plated onto 100-mm culture dishes (Costar ${ }^{\mathrm{TM}}$; Corning, Inc., Corning, NY, USA) were trypsinized and washed with FBS-free media and PBS. The cells were collected and fixed with $70 \%$ methanol at $4^{\circ} \mathrm{C}$. The cells were then treated with phosphate-citrate buffer for $5 \mathrm{~min}$. After centrifugation, the pellets were suspended in $300 \mu \mathrm{l}$ of $1 \%$ FBS-PBS and then treated with a final concentration of $100 \mu \mathrm{g} / \mathrm{ml} \mathrm{RNase} \mathrm{A}$ and $10 \mu \mathrm{g} / \mathrm{ml}$ propidium iodide at room temperature for $30 \mathrm{~min}$. After staining, 20,000 cells/sample were analyzed on a FACSCanto flow cytometer (Becton Dickinson Japan Co., Ltd., Tokyo, Japan).

SALL4 expression vector. The vector, inserted with the full-length SALL4 gene (pCMV6-SALL4), was purchased from Origene Technologies, Inc. (Rockville, MD, USA), and large-scale preparation was performed using the competent cells. A control vector (pCMV6-Mock) was constructed by digesting parental vector using AsiSI (SfaAI) and MluI, resulting in a lack of SALL4 sequence. Both the plasmids were transfected into cells using a Nucleofector II device and a Cell Line Nucleofector kit T (Lonza Japan, Ltd., Tokyo, Japan), according to the manufacturer's instructions.

Transduction of siRNA against SALL4. HP GenomeWide siRNA, designed to target the coding region (exon 2) of SALL4 (GenBank accession no. NM-020436), was purchased from Qiagen. Single-strand RNAs were annealed by incubating each strand in the siRNA suspension buffer at $90^{\circ} \mathrm{C}$ for $1 \mathrm{~min}$ and then at $37^{\circ} \mathrm{C}$ for $1 \mathrm{~h}$. Non-silencing control (NSC) RNA (Qiagen) was used as a transduction control. The transduction of siRNA was performed using the Nucleofector II device and the Cell Line Nucleofector kit T (Lonza Japan, Ltd.), according to the manufacturer's instructions.

In brief, approximately $2-4 \times 10^{6}$ cells were cultured under normal conditions to sub-confluency, and $1 \times 10^{6}$ cells were transduced with siRNA or NSC in a cuvette. Next, $5 \times 10^{4}$ of these transduced cells were plated on $3 \mathrm{ml}$ of medium supplemented with $10 \%$ FBS in a 6-well plate $\left(\right.$ Costar $^{\mathrm{TM}}$; Corning, Inc.). After 24-48 h, the expression of the silenced mRNA was quantified by TaqMan RT-PCR. The cells were collected at different periods and subjected to the cell viability assay and cell cycle analysis.

Statistical analysis. Data are presented as mean \pm SD. Statistical analysis was performed by paired Student's t-test and the Mann-Whitney rank sum test. $\mathrm{P}<0.05$ was considered to be significant.

\section{Results}

Effect of SALL4 siRNA transduction on drug sensitivity. To determine the role of SALL4 expression as a drug-resistant factor in cancer cells, we transduced SALL4 siRNA into lung cancer cells. In A549 and SBC-3 cells, the transduction of siRNA decreased the SALL4 mRNA expression level to $\sim 50 \%$ of the levels in NSC-transduced cells at $48 \mathrm{~h}$ after transduction (Fig. 1A and B). To use the cells with same extent of inhibitory effect on mRNA expression, different siRNA concentrations (200 nM for A549 cells and $100 \mathrm{nM}$ for SBC-3 cells) were used. Next, we determined how the transduction
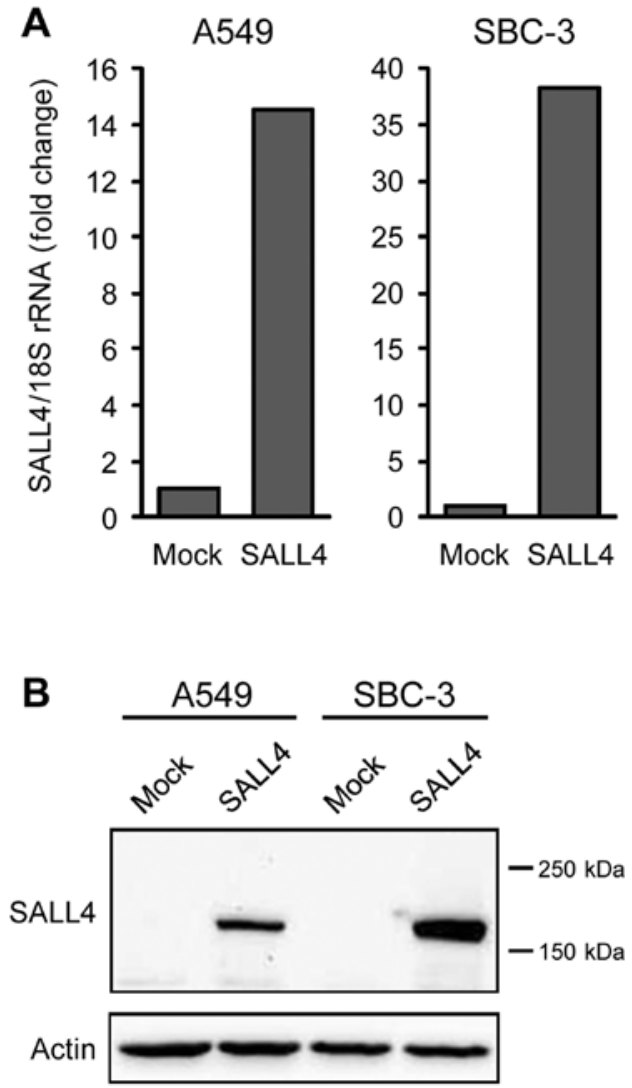

Figure 3. Alteration of SALL4 (A) mRNA and (B) protein expression in A549 and SBC-3 cells by transduction of pCMV6-SALL4. SALL4 mRNA expression level was measured by quantitative RT-PCR and is reported as a ratio relative to the amount in cells transduced with pCMV6-Mock set as 1.0. SALL4 protein expression was detected by western blotting.

of SALL4 siRNA affects drug sensitivity. We observed a slight decrease in growth at $48 \mathrm{~h}$ after transduction of NSC RNA and siRNA. After $48 \mathrm{~h}$ of siRNA transduction, the cells were treated with each anticancer drug. After treatment with CDDP, CBDCA, or PTX, the sensitivity of all anticancer drugs was significantly increased in siRNA-transduced cells. Drug sensitivity curves at day 4 after the treatment are shown in Fig. 2. The alteration of drug sensitivity was observed to be most potent in the PTX-treated cells and was also more potent in SBC-3 cells with relatively higher SALL4 mRNA expression, confirmed before starting the experiments. After treatment, the number of apparent apoptotic cells had not increased, and arrested growth and relatively larger cells were observed on day 4. In cell cycle analysis, the cell cycle pattern was observed to be altered in the cells treated with anticancer drugs after siRNA transduction; no increase in the sub-G1 fraction, reflecting apoptotic cells, was observed in any of the treatments (data not shown).

Microarray analysis of molecules regulated by SALL4. To examine the mechanism by which SALL4 regulates drug sensitivity, $\sim 65,000$ molecules on a microarray chip were analyzed using lung cancer cells transduced with a SALL4 expression vector (pCMV6-SALL4). In these cells, SALL4 mRNA and protein expression markedly increased at $24 \mathrm{~h}$ after transduction, unlike that in the cells transduced with the control vector (pCMV6-Mock) (Fig. 3A and B); these cells 
Table I. Molecules showing alteration of gene expression >2-fold change both in A549 and SBC-3 cells by microarray.

\begin{tabular}{lccl}
\hline Molecules & Log2 ratio (A549) & Log2 ratio (SBC-3) & \multicolumn{1}{c}{ Major molecular function } \\
\hline CSH-1/HPL & $4.81(28.1)^{\mathrm{a}}$ & $8.22(298.2)$ & $\begin{array}{l}\text { Placental development } \\
\text { Binding to mammary gland cell membrane } \\
\text { Growth of malignant tumors } \\
\text { IL-6 }\end{array}$ \\
TMEM229B & $1.43(2.69)$ & $1.25(2.38)$ & Transmission of survival signal via STAT3 \\
Ameloblastin & $2.26(4.78)$ & $2.61(6.10)$ & Unknown \\
Enamel formation of teeth
\end{tabular}

${ }^{a}$ Actual alteration is shown in parenthesis. CSH-1/HPL, chorionic somatomammotropin hormone-1/human placental lactogen; IL-6, interleukin-6; TMEM229B, transmembrane protein 229B.

Table II. Clinicopathologic backgrounds of the patients with lung cancer.

\begin{tabular}{|c|c|c|c|c|c|c|c|c|c|}
\hline $\mathrm{R}$ & No. & G & Age & Smoking & Stage & Type & Adjuvant chemotherapy & Days $^{\mathrm{a}}$ & Days $^{b}$ \\
\hline \multirow[t]{16}{*}{$(-)$} & 1 & M & 69 & Yes & IIIA & $\mathrm{SCC}$ & CDDP+PTX & N.A. & 874 \\
\hline & 2 & M & 64 & Yes & IIIA & SCC & $\mathrm{CBDCA}+\mathrm{PTX}$ & N.A. & 746 \\
\hline & 3 & $\mathrm{~F}$ & 71 & No & IIIA & Ad & $\mathrm{CBDCA}+\mathrm{PTX}$ & N.A. & 802 \\
\hline & 4 & M & 56 & Yes & IIA & $\mathrm{Ad}$ & CBDCA+PTX & N.A. & 748 \\
\hline & 5 & M & 63 & Yes & IIB & $\mathrm{SCC}$ & CBDCA+PTX & N.A. & 660 \\
\hline & 6 & $\mathrm{~F}$ & 66 & No & IA & Ad & UFT & N.A. & 562 \\
\hline & 7 & M & 65 & Yes & IA & SCLC & CDDP+CPT11 & N.A. & 1274 \\
\hline & 8 & M & 71 & Yes & IIA & SCLC & CPT11 & N.A. & 998 \\
\hline & 9 & M & 76 & Yes & IIIA & $\mathrm{Ad}$ & CBDCA+PEM & N.A. & 391 \\
\hline & 10 & M & 69 & Yes & IB & SCC & CBDCA+PTX & N.A. & 273 \\
\hline & 11 & M & 57 & Yes & IIIA & Ad & CBDCA+PEM & N.A. & 296 \\
\hline & 12 & M & 75 & Yes & IIIA & $\mathrm{SCC}$ & CBDCA+GEM & N.A. & 296 \\
\hline & 13 & $\mathrm{~F}$ & 61 & No & IB & Ad & UFT & N.A. & 1372 \\
\hline & 14 & M & 71 & Yes & IIB & $\mathrm{SCC}$ & CBDCA+PTX & N.A. & 1405 \\
\hline & 15 & M & 66 & Yes & IIIA & SCC & CDDP+VNR & N.A. & 1645 \\
\hline & 16 & M & 71 & Unknown & IIB & $\mathrm{SCC}$ & CBDCA+PTX & N.A. & 1807 \\
\hline \multirow[t]{15}{*}{$(+)$} & 17 & $\mathrm{~F}$ & 66 & Yes & IIIA & SCC & CDDP/CBDCA+CPT11 & 275 & N.A. \\
\hline & 18 & $\mathrm{~F}$ & 58 & Yes & IIA & Ad & CBDCA+PTX & 95 & N.A. \\
\hline & 19 & M & 67 & Yes & IB & SCLC & CBDCA+VP16 & 143 & N.A. \\
\hline & 20 & $\mathrm{~F}$ & 54 & Yes & IIIA & Ad & CBDCA+PEM/PEM & 44 & N.A. \\
\hline & 21 & M & 67 & Yes & IIIA & $\mathrm{SCC}$ & CBDCA/PTX & 151 & N.A. \\
\hline & 22 & M & 68 & Yes & IIIA & SCLC & $\mathrm{CBDCA}+\mathrm{VP} 16$ & 31 & N.A. \\
\hline & 23 & $\mathrm{~F}$ & 58 & No & IIIB & Ad & CBDCA+PEM & 139 & N.A. \\
\hline & 24 & M & 62 & Yes & IIIA & $\mathrm{SCC}$ & $\mathrm{CBDCA}+\mathrm{DOC}$ & 175 & N.A. \\
\hline & 25 & $\mathrm{~F}$ & 74 & No & IIIA & Ad & CBDCA+PTX & 308 & N.A. \\
\hline & 26 & $\mathrm{~F}$ & 51 & Yes & IIIA & Ad & $\mathrm{CBDCA}+\mathrm{PTX}$ & 735 & N.A. \\
\hline & 27 & M & 59 & Yes & IIB & $\mathrm{SCC}$ & CDDP+VNR & 181 & N.A. \\
\hline & 28 & M & 72 & Yes & IIIA & $\mathrm{SCC}$ & CBDCA+PTX & 410 & N.A. \\
\hline & 29 & M & 59 & Yes & IA & $\mathrm{SCC}$ & CBDCA+PEM/VP16 & 411 & N.A. \\
\hline & 30 & $\mathrm{~F}$ & 72 & Yes & IIB & Ad & CBDCA+PTX & 374 & N.A. \\
\hline & 31 & M & 49 & Yes & IIIA & Ad & CBDCA+PTX & 47 & N.A. \\
\hline
\end{tabular}

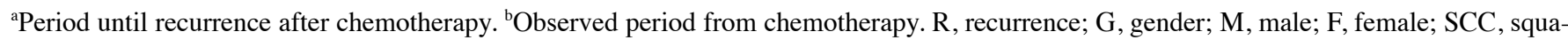
mous cell carcinoma; Ad, adenocarcinoma; SCLC, small cell lung carcinoma; CDDP, cisplatin; PTX, paclitaxel; CBDCA, carboplatin; UFT, tegafur-uracil; PEM, pemetrexed; GEM, gemcitabine; VNR, vinorelbine; DOC, docetaxel; N.A., not applicable.

were used for microarray analysis. Expression of numerous molecules was altered in A549 and SBC-3 cells, and molecules showing $>2$-fold change in expression in both cell types were taken to be candidate molecules. Only four molecules, 
chorionic somatomammotropin hormone-1/human placental lactogen (CSH-1/HPL), interleukin-6 (IL-6), transmembrane protein 229B (TMEM229B), and ameloblastin, met this criterion (Table I).

Expression of SALL4 mRNA in lung cancers. TaqMan RT-PCR was performed on cancerous tissue samples obtained from patients with lung cancer. The patient characteristics and clinical backgrounds are listed in Table II. First, the relationship between SALL4 mRNA expression and the clinicopathological backgrounds were analyzed (Table III). The mean value was affected by one case showing extremely high expression; however, gender, age, pathologic type, and pathologic stage showed no statistically significant effect. On the contrary, in the group showing recurrence of cancer after chemotherapy, the SALL4 mRNA level was significantly higher than that in the group without recurrence (Table III, $\mathrm{p}=0.031$ ). The mean SALL4 mRNA expression level in the group showing recurrence $(125.0 \pm 318.5)$ was 34 -fold higher than that in the group without recurrence (3.7 \pm 9.2$)$ (Fig. 4). Even when four patients with small cell lung carcinoma were excluded, the group with recurrence showed significantly higher expression $(139.8 \pm 341.4)$ compared to the expression $(1.1 \pm 2.9)$ in the group without recurrence $(\mathrm{p}<0.05)$. When the cut-off value was set at the mean $\pm 2 \mathrm{SD}$ in cases without recurrence, cases showing positive expression ( $8 / 15$ cases) showed a shorter period until recurrence $(177 \pm 117$ days) compared to that observed in the seven negative cases ( $300 \pm 242$ days). Even when two patients with small cell lung carcinoma were excluded, the period until recurrence was shorter (208 \pm 106 days) in the positive cases.

\section{Discussion}

We have previously reported that $S A L L 4$, a gene essential for stem cell replication, showed an upregulated expression in the cancerous cells than in the non-cancerous cells in lung cancer patients $(19,20)$. However, its clinical significance, other than as a marker to support the diagnosis of cancer, has not been determined. In the present study, we report the first evidence that SALL4 expression could be a resistance factor against anticancer drugs in lung cancer. The majority of recent studies investigating the factors that determine drug sensitivity in lung cancer have focused on the expression or mutation of target molecules such as EGFR, K-ras, and EML4/ALK preceding molecular target therapy. However, no markers that can be put to clinical use have yet been developed for the factors regulating the sensitivity of conventional chemotherapeutic drugs such as CDDP, CBDCA, and PTX. For example, the anti-apoptotic molecule bcl-2 has been reported to produce resistance to anticancer drugs when overexpressed in lung cancer cells (22), leading to the development of a bcl-2 inhibitor to increase drug sensitivity. However, bcl-2 expression has not yet been sufficiently analyzed in clinical samples, and therefore its significance as a prognostic factor remains uncertain. Although the sample numbers are rather small and a definitive conclusion cannot be reached, the results of the present study clarify that SALL4 expression before therapy tends to be higher in cases resulting in recurrence after adjuvant chemotherapy. In addition, the results showed that the period until recurrence is shorter in cases showing higher
Table III. Relationship between SALL4 mRNA expression and clinicopathologic backgrounds.

\begin{tabular}{lrrrr}
\hline Factors & No. & Mean & SD & P-value \\
\hline Gender & & & & \\
$\quad$ Male & 21 & 16.8 & 29.4 & 1.000 \\
$\quad$ Female & 10 & 158.1 & 391.9 & \\
Age & & & & \\
$\quad<60$ years old & 9 & 36.2 & 75.5 & 0.457 \\
$\quad \geq 60$ years old & 22 & 73.1 & 265.5 & \\
Pathologic diagnosis & & & & \\
Ad & 13 & 25.1 & 64.1 & 0.207 \\
SCC & 14 & 107.5 & 332.0 & \\
SCLC & $4^{\mathrm{a}}$ & 25.8 & 12.1 & \\
Stage & & & & \\
I+II & 14 & 15.0 & 31.8 & 0.892 \\
III & 17 & 101.5 & 302.5 & \\
Prognosis & & & & \\
$\quad$ Desease-free & 16 & 3.7 & 9.2 & 0.031 \\
Reccurence & 15 & 125.0 & 318.5 & \\
\hline
\end{tabular}

$\mathrm{P}<0.05$ was considered to be significant by Mann-Whitney rank sum

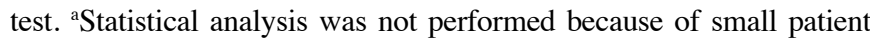
numbers. SCC, squamous cell carcinoma; Ad, adenocarcinoma; SCLC, small cell lung carcinoma.

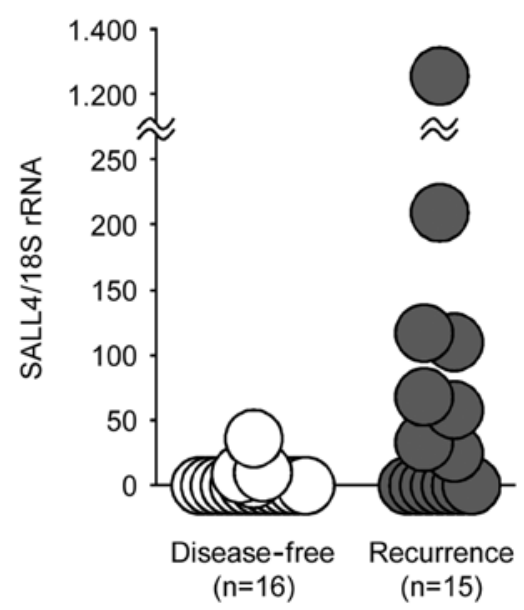

Figure 4. Comparison of pre-therapy levels of SALL4 mRNA expression between cases that did or did not recur after adjuvant chemotherapy. SALL4 mRNA levels were measured using quantitative RT-PCR and are expressed relative to the amount of $18 \mathrm{~S}$ rRNA. Statistical analysis was performed using the Mann-Whitney rank sum test. Expression differed significantly between disease-free and recurrent cases $(\mathrm{p}=0.031)$.

SALL4 expression. These results indicate that measurement of SALL4 expression may be useful to estimate the existence of very small amounts of residual cancer cells, which cannot be detected by conventional computed tomography (CT) or magnetic resonance imaging (MRI), after surgery.

Comparison of recurring and non-recurring cases showed no bias on the basis of the type of cancer drugs used; both 
platinum drugs and taxol are widely used. Further, an in vitro sensitivity test of SALL4 inhibition showed altered sensitivity to all types of anticancer drugs. Taken together with data from clinical samples, it appears that SALL4 might be a universal resistance factor against anticancer drugs.

In the present study, in cases where SALL 4 siRNA increased the sensitivity, the in vitro concentration of anticancer drugs used was less than the concentration measurable in the blood after administration of a clinical dose. This suggests that SALL4 inhibition could augment cancer-cell sensitivity to relatively lower concentrations of both platinum drugs and taxol, of which dosage cannot be increased because of adverse effects, such as gastrointestinal toxicity, renal toxicity, and decreased platelet counts. In addition, both non-small cell carcinoma of A549 cells and small cell SBC-3 cells showed increased drug sensitivity, suggesting that SALL4 could be target molecules for augmenting drug sensitivity, regardless of the cancer type.

A previous study has shown that the drug transporters ABCG2 and ABCA3 can be induced by SALL4 in leukemic cells (21). However, our microarray analysis using SALL4-overexpressed lung cancer cells did not reveal any alteration in the expression levels of either of these mRNAs (data not shown). In this experiment, four molecules showed elevated expression in both examined cell lines. In these candidates, molecular function of CSH-1/HPL, TMEM229B, and ameloblastin in cancer cells have not been previously reported. Only IL-6 is known to transmit growth and survival signals via STAT3 activation in lung cancer cells, and SALL4 expression is reported to be upregulated by STAT3 (23-25), suggesting that the STAT3 pathway may be involved in drug resistance and that a positive-feedback loop may exist between SALL4 and IL-6 via STAT3. Further study may prove this speculation and clarify the molecular significance of CSH-1/HPL, TMEM229B, and ameloblastin.

Molecules regulating stem cell replication are known to show reciprocal augmentation of gene expression. SALL4 and Nanog, a factor which maintains the undifferentiated state of stem cells, have been reported to show reciprocally augmented gene expression in mouse ES cells (26). In the preliminary experiment, we recently found that SALL4 expression vector-transduced cells showed an increase in Nanog mRNA expression (data not shown). The molecular function of Nanog in cancer cells remains unclear, but it is possible that SALL4 and Nanog cooperatively form a fundamental feature for maintaining the undifferentiated state and promotion of cell proliferation.

The results of the present study clarify that SALL4 acts as a constitutive resistance factor against anticancer drugs and suggest that recurrence after adjuvant chemotherapy could be predicted in lung cancer cases showing overexpression of SALL4 before therapy. In addition, because both the combination of SALL4 inhibition and anticancer drugs, and siRNA alone can produce remarkable inhibition of cell proliferation in some cell lines (20), SALL4 shows promise as a novel therapeutic target.

The involvement of SALL4 in acquired resistance remains unclear. We preliminarily examined SALL4 expression in cells cultured under conditions of step-wise increase of CDDP concentration. SALL4 expression increased for several weeks but then decreased to the level of constitutive expression. This observation suggests the involvement of SALL4 in stress response, but its significance as a factor for acquired resistance remains to be investigated.

\section{Acknowledgements}

This study was supported by the grants from Japan Society for the Promotion of Science (Tokyo, Japan).

\section{References}

1. Pardal R, Molofsky AV, He S and Morrison SJ: Stem cell self-renewal and cancer cell proliferation are regulated by common networks that balance the activation of proto-oncogenes and tumor suppressors. Cold Spring Harb Symp Quant Biol 70: $177-185,2005$.

2. Yasuda SY, Tsuneyoshi N, Sumi T, Hasegawa K, Tada T, Nakatsuji N and Suemori H: NANOG maintains self-renewal of primate ES cells in the absence of a feeder layer. Genes Cells 11: 1115-1123, 2006.

3. Zhou Q, Chipperfield H, Melton DA and Wong WH: A gene regulatory network in mouse embryonic stem cells. Proc Natl Acad Sci USA 104: 16438-16443, 2007.

4. Zhang J, Tam WL, Tong GQ, et al: Sall4 modulates embryonic stem cell pluripotency and early embryonic development by the transcriptional regulation of Pou5f 1 . Nat Cell Biol 8: 1114-1123, 2006.

5. Jiang J, Chan YS, Loh YH, et al: A core Klf circuitry regulates self-renewal of embryonic stem cells. Nat Cell Biol 10: 353-360, 2008.

6. Okita K, Ichisaka T and Yamanaka S: Generation of germlinecompetent induced pluripotent stem cells. Nature 448: 313-317, 2007.

7. Tsubooka N, Ichisaka T, Okita K, Takahashi K, Nakagawa M and Yamanaka S: Roles of Sall4 in the generation of pluripotent stem cells from blastocysts and fibroblasts. Genes Cells 14: 683-694, 2009.

8. Yu J, Vodyanik MA, Smuga-Otto K, et al: Induced pluripotent stem cell lines derived from human somatic cells. Science 318: 1917-1920, 2007.

9. Sakaki-Yumoto M, Kobayashi C, Sato A, et al: The murine homolog of SALL4, a causative gene in Okihiro syndrome, is essential for embryonic stem cell proliferation, and cooperates with Sall1 in anorectal, heart, brain and kidney development. Development 133: 3005-3013, 2006.

10. Jürgens G: Head and tail development of the Drosophila embryo involves spalt, a novel homeotic gene. EMBO J 7: 189-196, 1998.

11. Al-Baradie R, Yamada K, St Hilaire C, et al: Duane radial ray syndrome (Okihiro syndrome) maps to $20 \mathrm{q} 13$ and results from mutations in SALL4, a new member of the SAL family. Am J Hum Genet 71: 1195-1199, 2002.

12. Kohlhase J, Heinrich M, Liebers M, et al: Cloning and expression analysis of SALL4, the murine homologue of the gene mutated in Okihiro syndrome. Cytogenet Genome Res 98: 274-277, 2002.

13. Ma Y, Cui W, Yang J, et al: SALL4, a novel oncogene, is constitutively expressed in human acute myeloid leukemia (AML) and induces AML in transgenic mice. Blood 108: 2726-2735, 2006.

14. Cui W, Kong NR, Ma Y, Amin HM, Lai R and Chai L: Differential expression of the novel oncogene, SALL4, in lymphoma, plasma cell myeloma, and acute lymphoblastic leukemia. Mod Pathol 19: 1585-1592, 2006

15. Yang J, Chai L, Gao C, et al: SALL4 is a key regulator of survival and apoptosis in human leukemic cells. Blood 112: 805-813, 2008.

16. Yang J, Chai L, Liu F, et al: Bmi-1 is a target gene for SALL4 in hematopoietic and leukemic cells. Proc Natl Acad Sci USA 104: 10494-10499, 2007.

17. Dimri GP, Martinez JL, Jacobs JJ, et al: The Bmi-1 oncogene induces telomerase activity and immortalizes human mammary epithelial cells. Cancer Res 62: 4736-4745, 2002.

18. Liu L, Andrews LG and Tollefsbol TO: Loss of the human polycomb group protein BMI1 promotes cancer-specific cell death. Oncogene 25: 4370-4375, 2006. 
19. Kobayashi D, Kuribayashi K, Tanaka M and Watanabe $\mathrm{N}$ : SALL4 is essential for cancer cell proliferation and is overexpressed at early clinical stages in breast cancer. Int J Oncol 38 : 933-999, 2011.

20. Kobayashi D, Kuribayashi K, Tanaka M and Watanabe N: Overexpression of SALL4 in lung cancer and its importance in cell proliferation. Oncol Rep 26: 965-970, 2011.

21. Jeong HW, Cui W, Yang Y, Lu J, He J, Li A, Song D, Guo Y, Liu BH and Chai L: SALL4, a stem cell factor, affects the side population by regulation of the ATP-binding cassette drug transport genes. PLoS One 6: e18372, 2011.

22. Abou El Hassan MA, Mastenbroek DC, Gerritsen WR, Giaccone G and Kruyt FA: Overexpression of Bcl2 abrogates chemo- and radiotherapy-induced sensitisation of NCI-H460 non-small-cell lung cancer cells to adenovirus-mediated expression of full-length TRAIL. Br J Cancer 91: 171-177, 2004.
23. Yi H, Cho HJ, Cho SM, Jo K, Park JA, Kim NH, Amidon GL, Kim JS and Shin HC: Blockade of interleukin-6 receptor suppresses the proliferation of H460 lung cancer stem cells. Int J Oncol 41: 310-316, 2012.

24. Song L, Rawal B, Nemeth JA and Haura EB: JAK1 activates STAT3 activity in non-small-cell lung cancer cells and IL-6 neutralizing antibodies can suppress JAK1-STAT3 signaling. Mol Cancer Ther 10: 481-494, 2011.

25. Bard JD, Gelebart P, Amin HM, Young LC, Ma Y and Lai R: Signal transducer and activator of transcription 3 is a transcriptional factor regulating the gene expression of SALL4. FASEB J 23: 1405-1414, 2009.

26. Wu Q, Chen X, Zhang J, et al: Sall4 interacts with Nanog and co-occupies Nanog genomic sites in embryonic stem cells. J Biol Chem 281: 24090-24094, 2006. 